Z Epileptol $2011 \cdot 24: 85-86$

DOI 10.1007/s10309-011-0166-x

Online publiziert: 30. April 2011

(c) Springer-Verlag 2011

\author{
H. Lerche ${ }^{1}$ - B. Neubauer ${ }^{2}$ \\ ${ }^{1}$ Abt. Neurologie mit Schwerpunkt Epileptologie, Zentrum für Neurologie, \\ Hertie Institut für Klinische Hirnforschung, Universität Tübingen, Tübingen \\ ${ }^{2}$ Abteilung Neuropädiatrie, Sozialpädiatrie und Epileptologie, \\ Universitäts-Kinderklinik Gießen, Gießen
}

\section{Genetische Untersuchungen bei Epilepsien}

\section{Ein neues Zeitalter beginnt}

Etwa 40\% der Epilepsien haben überwiegend genetische Ursachen. Es handelt sich dabei v. a. um die große Gruppe der idiopathischen Epilepsien, die keine strukturellen Hirnläsionen aufweisen und über die jetzt diskutiert wird, sie in „genetische Epilepsien“ umzubenennen [1]. Bei diesen Krankheiten bestehen meist Veränderungen der genomischen DNA, die in allen Zellen des Körpers, auch der Keimbahn, vorhanden sind und damit vererbt werden. Selten treten Mosaike auf, die durch Mutationen während der Entwicklung zustande kommen und nur einen Teil der Körperzellen betreffen. Zudem kann die mitochondriale DNA betroffen sein. Man unterscheidet monogenetische Ursachen, die allein ausreichend sind, um die Krankheit hervorzurufen, von genetischen Suszeptibilitätsfaktoren, die in unterschiedlichem Maß die Wahrscheinlichkeit erhöhen, dass jemand an Epilepsie erkrankt. Monogenetisch verursachte Epilepsien sind selten (1-2\% der idiopathischen Epilepsien), während die häufigsten Formen der primär genetisch bedingten Epilepsien, die idiopathischen generalisierten Epilepsien (IGE) und die Rolando-Epilepsie (bzw. zentrotemporale „spikes“), einem komplex-genetischen Erbgang folgen. Darunter wird verstanden, dass mehrere genetische Ursachen, die unterschiedlich stark für die Erkrankung ver- antwortlich sind, zusammenkommen müssen, damit eine Epilepsie entsteht. Der Übergang von monogenetischen Ursachen über sog. Hauptgeneffekte mit einer großen Auswirkung auf den Phänotyp bis hin zu Suszeptibilitätsfaktoren, die die Wahrscheinlichkeit, an Epilepsie zu erkranken, vielleicht nur um 5-10\% erhöhen, ist möglicherweise fließend.

Die meisten bisher bekannten genetischen Veränderungen wurden bei monogenetischen Epilepsien gefunden, weil sie dort am einfachsten über klassische Kopplungsanalysen und anschließende Kandidatengensequenzierung zu entdecken sind. Die zunehmende Verfügbarkeit genomweiter Techniken, von der Assoziationsanalyse über die Bestimmung der Kopienanzahl bestimmter Genregionen („,copy number variations“, CNV) bis hin zur jetzt seit Kurzem verfügbaren Sequenzierung der gesamten codierenden DNA („whole exome sequencing“) und schließlich der genomweiten Sequenzierung, erlaubt nun die annähernd komplette Entschlüsselung der genetischen Faktoren von Epilepsien. Hier stehen wir tatsächlich am Beginn einer neuen Epoche. Fragestellungen, deren Klärung vor Kurzem noch Jahre gedauert hätte, lassen sich heute durch direkte Sequenzierung in Monaten bearbeiten. Als zweitgrößtes "chapter“ der International League Against Epilepsy (ILAE) haben wir jetzt in der deutschen Epileptologie die Chance und die Möglichkeiten, das Feld in unserem Fach entscheidend voranzubringen.

Die bisher wichtigsten Befunde betreffen Mutationen in verschiedenen Ionenkanalgenen, die die Erregbarkeit des Gehirngewebes verändern [8], und die kürzlich gefundenen Veränderungen der CNV an bestimmten chromosomalen Loci, die einen wichtigen Risikofaktor für die IGE $([3,5,7])$ und weniger wahrscheinlich auch für symptomatische oder kryptogene fokale Epilepsien ausmachen [4], ohne dass der Mechanismus bisher verstanden ist.

Neben diesen Veränderungen, die eine Epilepsie bedingen können, werden zunehmend pharmakogenetische Aspekte diskutiert und erforscht. Die Fragen, die sich hierbei stellen, sind „Warum reagiert ein Patient gut und ein anderer sehr schlecht auf Medikamente?" und „Warum entwickelt ein Patient Nebenwirkungen und ein anderer nicht?". Hier könnten genetische Faktoren eine sehr wichtige Rolle spielen. Als Beispiel soll ein genetischer Risikofaktor in einem „Human-leukocyte-antigen“- (HLA-) Gen für schwere allergische Hautreaktionen auf Carbamazepin in der südostasiatischen Bevölkerung genannt sein, für den jetzt der Nutzen einer klinisch-prädiktiven Anwendung eindrucksvoll be- 
wiesen wurde [2]. Auch für die kaukasische Bevölkerung konnte nun ein solcher Risikofaktor in einem anderen HLA-Gen nachgewiesen werden [6]. Neben den primären Veränderungen der DNA-Sequenz spielen wahrscheinlich auch sekundäre Veränderungen eine sehr wichtige Rolle für Epilepsien, wie z. B. die Methylierung oder die Acetylierung der DNA, die unter dem Begriff „Epigenetik“ zusammengefasst werden. Sie könnten bei der Entstehung sowohl erworbener als auch vererbter Epilepsiesydrome von Belang sein und sich auch im Laufe der Behandlung verändern.

Die vorliegende Ausgabe der Zeitschrift für Epileptologie widmet sich der gesamten Bandbreite der oben genannten genetischen Faktoren, deren Einfluss auf die Entstehung von Epilepsien und die Modifikation des Krankheitsverlaufs. Dabei wird sowohl auf praktische Belange eingegangen, die bereits heute im klinischen Alltag wichtig sind, als auch auf die wissenschaftlichen Aspekte. Wir möchten mit der Ausgabe nicht nur informieren und das Interesse für die genetischen Krankheitsmechanismen wecken, sondern auch ganz konkret alle, die mit Epilepsiepatienten zu tun haben, zur Mitarbeit bewegen. Die Grundlage für alle genetischen Untersuchungen sind klinisch gut charakterisierte Patienten. Deshalb steht die Rekrutierung von geeigneten Patienten immer an erster Stelle jedes genetischen Projekts. In Deutschland haben bereits viele zu den bisherigen Erfolgen der Genetik beigetragen, in dem sie Patienten von solchen Untersuchungen überzeugt und sie an forschende Einrichtungen vermittelt haben. Es hat sich gezeigt, dass $\mathrm{z}$. T. sehr große $\mathrm{Pa}$ tientenzahlen erforderlich sind, um die oben aufgeworfenen Fragen umfassend zu lösen. Insbesondere Faktoren, die nur kleine Effekte haben, brauchen naturgemäß eine hohe Zahl von Patienten, um ihre statistische Signifikanz nachzuweisen. Deshalb werden diese Fragen in größeren Forschungskonsortien bearbeitet. $\mathrm{Zu}$ nennen sind v. a. das nationale $\mathrm{Ge}$ nomforschungsnetz (www.nfgn.de), das durch das Bundesministerium für Bildung und Forschung seit vielen Jahren gefördert wird und das weiterläuft, sowie das erste größere europäische Epilep- sieprojekt EPICURE (www.epicureproject.eu). Diese Projekte haben zur größten Sammlung von DNA-Proben bei IGE weltweit und auch zur größten Bank von epilepsiechirurgisch gewonnenem Gewebe geführt (www.epilepsie-register. de). Den Autoren der Beiträge in dieser Ausgabe ist es nun gelungen, ein weiteres europäisches Projekt zu verschiedenen Aspekten der Epilepsiegenetik einzuwerben. Es geht v. a. um die Identifikation von Gendefekten mit den neu zur Verfügung stehenden Hochdurchsatztechniken. Es handelt es sich um ein Programm der European Science Foundation (ESF), das sich aus vielen nationalen Förderungen in europäischer Kooperation zusammensetzt. In Deutschland ist dafür die Deutsche Forschungsgemeinschaft (DFG) zuständig. Ganz konkret sind wir für diese Projekte an der Vermittlung von Patienten mit folgenden Krankheiten interessiert:

- seltene, monogenetische Epilepsiesyndrome,

- epileptische Enzephalopathien,

- IGE,

- Rolando-Epilepsien und verwandte Syndrome bzw. zentrotemporale „spikes“ im EEG,

- Temporallappenepilepsien und

- Patienten für pharmakogenetische Untersuchungen.

Genauere Informationen zu den Kriterien für geeignete Patienten finden sich in den jeweiligen Beiträgen. Dabei geht es sowohl um die Vermittlung einzelner Familien oder Patienten als auch um Kooperationen, bei denen an interessierten Stellen eigenständig rekrutiert wird.

Die Zusammenarbeit von Klinikern und genetisch interessierten Wissenschaftlern hat sich in der Vergangenheit als sehr erfolgreich für beide Seiten dargestellt, da auch die weitere Bearbeitung klinischer Aspekte durch die Sammlung solcher Patientenkohorten gefördert wird. Die Beschreibung von neu entdeckten oder Varianten bekannter Syndrome, Fragen der Genotyp-Phänotyp-Beziehungen, der Endophänotypisierung und assoziierter Krankheiten (z. B. Epilepsie und Migräne) sind hier beispielhaft zu nennen. Einige Leser werden auch eigene Fragen zu genetischen Untersuchun- gen bei ihren Patienten haben, für die wir gern Hilfestellung anbieten oder als Kooperationspartner zur Verfügung stehen.

Die Autoren danken Ihnen im Voraus für Ihr Interesse und Ihre Mitarbeit.

Holger Lerche und Bernd Neubauer

\section{Korrespondenzadresse \\ Prof. Dr. H. Lerche}

Abt. Neurologie mit Schwerpunkt Epileptologie, Zentrum für Neurologie, HertieInstitut für Klinische Hirnforschung,

Universität Tübingen

Hoppe-Seyler-Str. 3, 72076 Tübingen

holger.lerche@uni-tuebingen.de

\section{Korrespondenzadresse}

\section{Prof. Dr. B. Neubauer}

Abteilung Neuropädiatrie, Sozialpädiatrie und Epileptologie, Universitäts-Kinderklinik Gießen Feulgenstr. 10-12, 35385 Gießen

Bernd.A.Neubauer@paediat.

med.uni-giessen.de

Interessenkonflikt. Die korrespondierenden

Autoren gibt an, dass kein Interessenkonflikt besteht.

\section{Literatur}

1. Berg AT, Berkovic SF, Brodie MJ et al (2010) Revised terminology and concepts for organization of seizures and epilepsies: report of the ILAE Commission on Classification and Terminology, 20052009. Epilepsia 51:676-685

2. Chen P, Lin JJ, Lu CS et al (2011) Carbamazepineinduced toxic effects and HLA-B*1502 screening in Taiwan. N Engl J Med 364:1126-1133

3. de Kovel CG, Trucks H, Helbig I et al (2010) Recurrent microdeletions at 15q11.2 and 16p13.11 predispose to idiopathic generalized epilepsies. Brain 133:23-32

4. Heinzen EL, Radtke RA, Urban TJ et al (2010) Rare deletions at $16 \mathrm{p} 13.11$ predispose to a diverse spectrum of sporadic epilepsy syndromes. Am J Hum Genet 86:707-718

5. Helbig I, Mefford HC, Sharp AJ et al (2009) $15 q 13.3$ microdeletions increase risk of idiopathic generalized epilepsy. Nat Genet 41:160-162

6. McCormack M, Alfirevic A, Bourgeois $S$ et al (2011) HLA-A*3101 and carbamazepine-induced hypersensitivity reactions in Europeans. N Engl J Med 364:1134-1143

7. Mefford HC, Muhle H, Ostertag P, et al. (2010) Genome-wide copy number variation in epilepsy: novel susceptibility loci in idiopathic generalized and focal epilepsies. PLoS Genet 6:e1000962

8. Weber YG, Lerche H (2008) Genetic mechanisms in idiopathic epilepsies. Dev Med Child Neurol 50:648-654 